\title{
Evaluation of differences in the expression of TNF, TNFR1, TNFR2 and dermatological scales under conventional and anti-cytokine therapy of psoriasis
}

Beniamin Oskar Grabarek ${ }^{1}$, Dominika Wcisło-Dziadecka², Jakub Krzaczyński1 ${ }^{1}$, Agnieszka Gancarczyk ${ }^{2}$, Anita Lyssek-Boroń $^{3,4}$

${ }^{I}$ Department of Histology, Cytophysiology and Embryology, Faculty of Medicine in Zabrze, University of Technology in Katowice,

Zabrze, Poland

${ }^{2}$ Department of Cosmetology, Faculty of Pharmaceutical Sciences in Sosnowiec, Medical University of Silesia in Katowice, Poland

Department of Ophthalmology with Paediatric Unit, $5^{\text {th }}$ Regional Hospital, Medykow Square 1, Sosnowiec, Poland

${ }^{4}$ Department of Ophtamology, Faculty of Medicine in Zabrze, University of Technology in Katowice, Poland

\section{INTRODUCTION}

- Pharmacotherapy of psoriasis vulgaris and arthritis includes using biological drugs (adalimumab, etanercept - anti TNF- $\alpha$, ustekinumab anti-IL 12/23) or conventional drugs, i.e. cyclosporine A.

- Unfortunately, during this treatment, the drug-resistance phenomenon was observed and the search for a new class of molecular markers is mandatory.

\section{AIM}

The aim of this study was to evaluate changes in the expression profile of tumor necrosis factor alpha (TNF- $\alpha$ ) and its receptor - TNFR1 and TNFR2 in psoriatic patients during adalimumab, etanercept, ustekinumab or cyclosporine A therapy compared with a group of healthy volunteers and to search for a relationship between molecular markers and clinical scales of treatment effectiveness - PASI, BSA, DAS28, DLQI and keratinocyte cell cultures.

\section{MATERIAL AND METHODS}
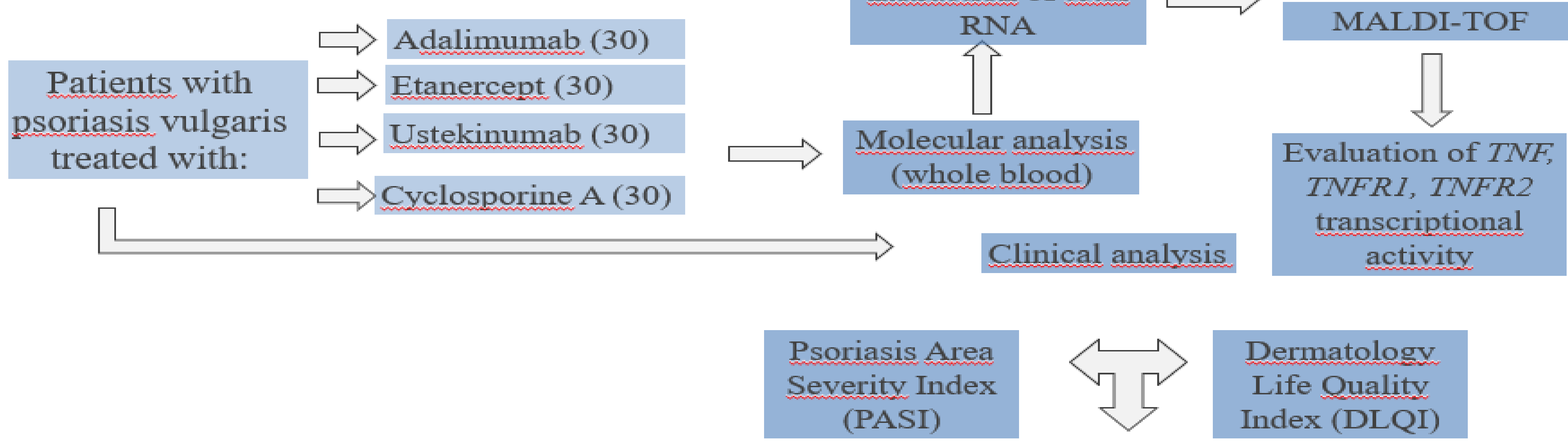

\section{Body Surface $\quad$ Disease Activity

\section{RESULTS}

\section{CONCLUSSION}
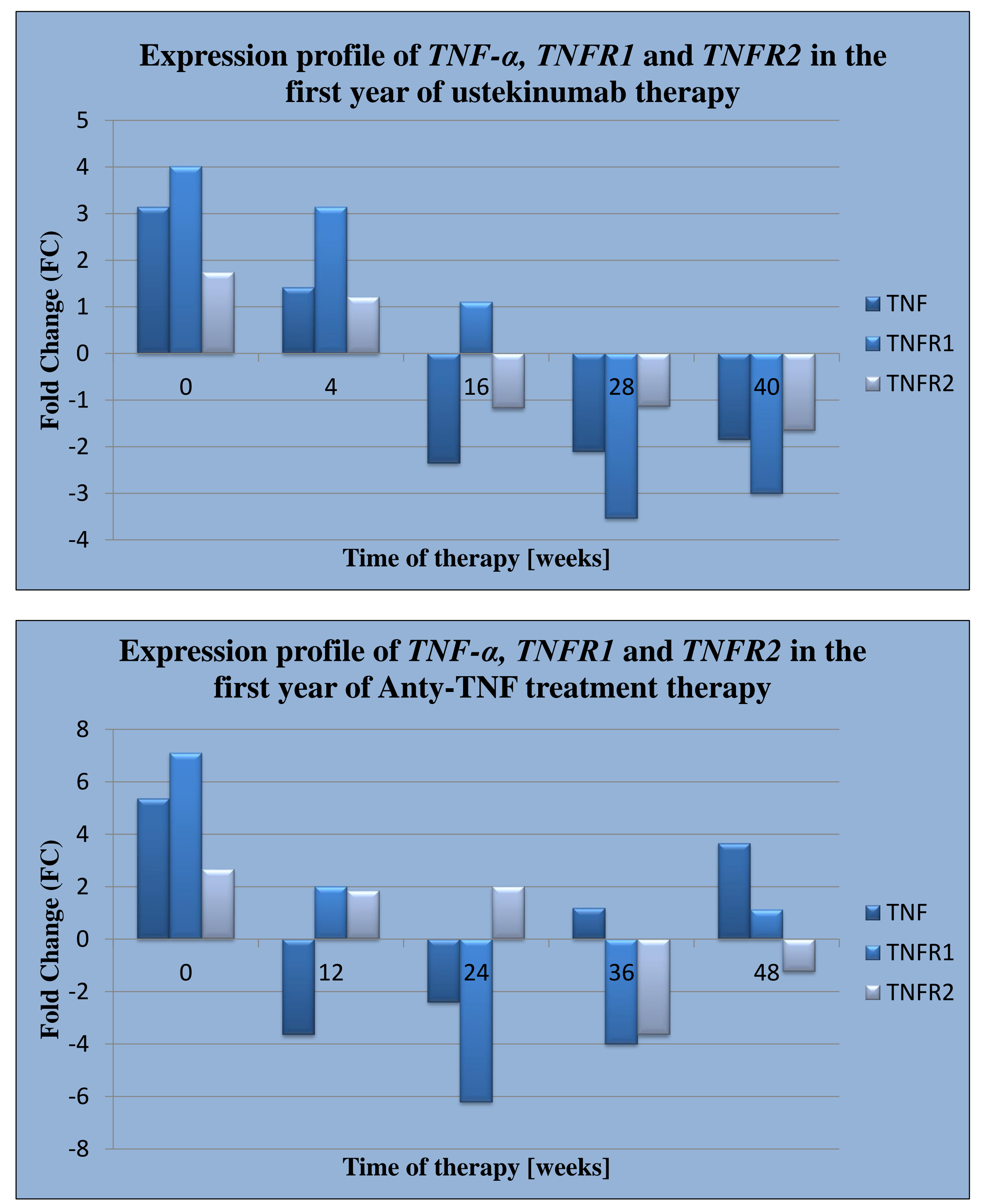
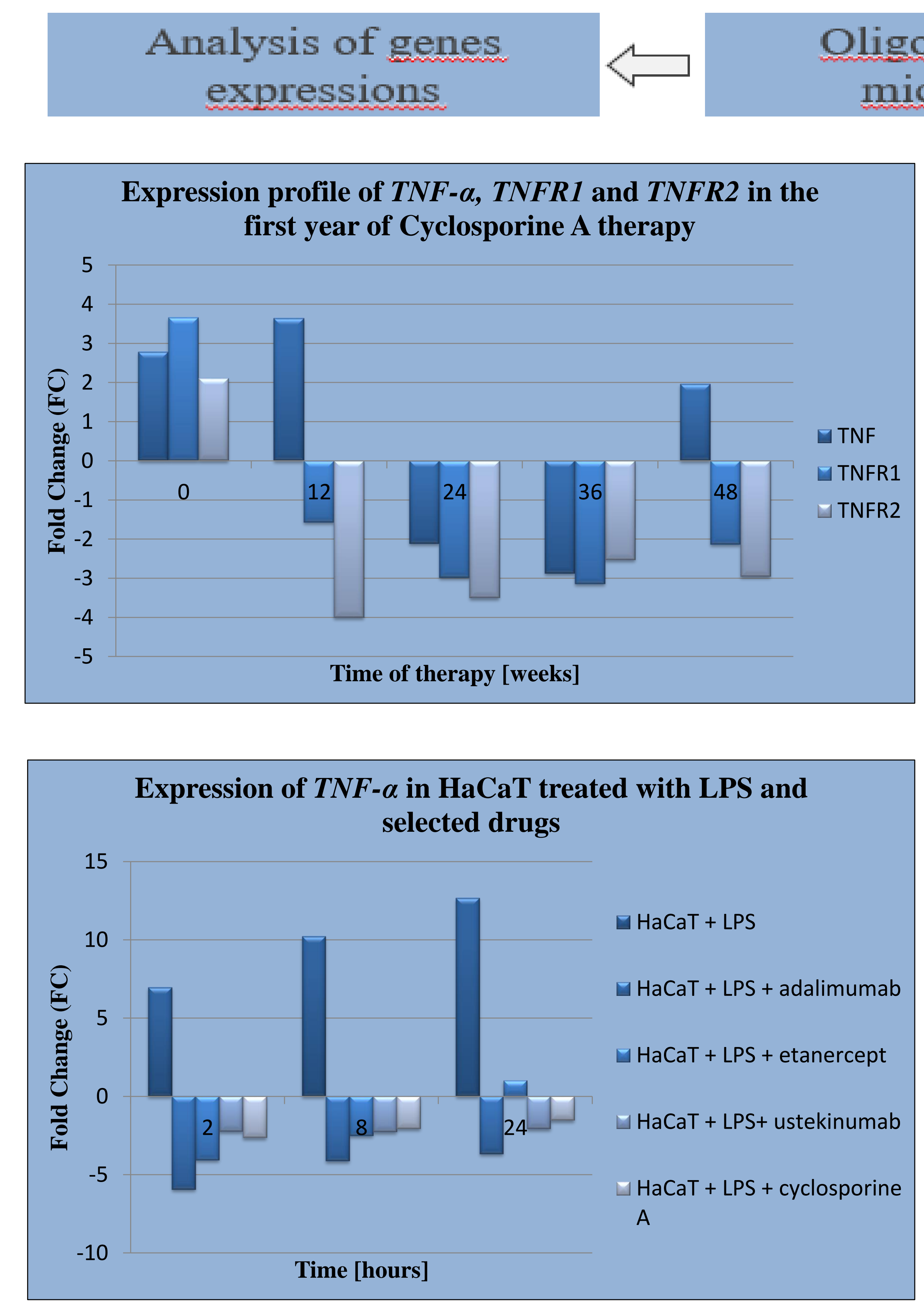

- $\quad$ TNF- $\alpha$ and its receptors seem to be useful molecular markers of psoriasis therapy effectiveness. 